\title{
NFC como alternativa para mejorar el desarrollo de videojuegos en dispositivos móviles
}

\author{
Stiven Muñoz-Quintero* \\ Juan Rincón Patino** \\ Gustavo Ramirez-Gonzalez ${ }^{* * *}$
}

Recibido: 29/07/2015 - Aceptado: 11/12/2015

DOI: 10.22395/rium.v15n28a8

\begin{abstract}
Resumen
La aparición de nuevas alternativas de interacción puede llevar a generar videojuegos cada vez más interesantes; este es el caso de la interacción con el teléfono móvil, especialmente la tecnología NFC. Sin embargo, no existe un referente para conocer qué tipos de videojuegos serían los más apropiados para desarrollar con NFC. Por tanto, este artículo aporta una revisión de varias fuentes para determinar el estado actual de videojuegos con NFC para futuras referencias de generadores de contenido y emprendedores en el campo de los videojuegos; como contribuciones se tiene una revisión en varias fuentes de nivel comercial, bibliográfico y de tiendas de aplicaciones para generar el estado actual y el esquema de posibles videojuegos con los cuales NFC podría interactuar, determinando las categorías de videojuegos más apropiadas para desarrollar con dicha tecnología y proponiendo algunas características que NFC permite implementar.
\end{abstract}

Palabras clave: NFC, videojuegos, dispositivos móviles

Estudiante de Ingeniería Electrónica y Telecomunicaciones en la Universidad del Cauca, Colombia. Carrera 2 \# 4N-140, Popayán, Colombia. Tel. +57 (8) 209800 Ext. 2127. stivenmunoz@unicauca.edu.co

** Estudiante de Ingeniería Electrónica y Telecomunicaciones en la Universidad del Cauca, Colombia. Carrera 2 \# 4N-140, Popayán, Colombia. Tel. +57 (8) 209800 Ext. 2127. juanrincon@unicauca.edu.co

*** Doctor en Ingeniería Telemática, Universidad Carlos III, España. M. Sc. en Ingeniería Telemática, Universidad del Cauca, Colombia. Ingeniero en Electrónica y Telecomunicaciones, Universidad del Cauca, Colombia. Docente e Investigador en el Departamento de Telemática de la Universidad del Cauca. Carrera 2 \# 4N-140, Popayán, Colombia. Tel.+57 (8) 209800 Ext. 2127. gramirez@unicauca.edu.co 


\title{
NFC as an Alternative for Improving Videogame Development in Mobile Devices
}

\begin{abstract}
The emergence of new interaction alternatives can result in increasingly interesting videogames; this is the case of the interaction with the mobile phone, especially NFC technology. However, there is not a model to get to know the types of videogames that would be more appropriate to be developed with NFC. Therefore, this article provides a review of several sources to determine current state of videogames with NFC for future reference of content generators and entrepreneurs in the field of videogames; as a contribution, there is a review of several commercial, bibliographic sources and applications shops to generate current state and a scheme of potential videogames with which NFC could interact, by determining categories of the most appropriate videogames to be developed with such a technology and proposing some features that can be implemented with the NFC.
\end{abstract}

Key words: NFC; videogames; mobile devices. 


\section{INTRODUCCIÓN}

El siguiente artículo presenta la investigación al nivel de vigilancia, realizada para conocer el uso de la tecnología Near Field Communication (NFC, Comunicación de Campo Cercano) en los videojuegos para dispositivos móviles; en este se muestra la búsqueda realizada en fuentes de información comercial, en las tiendas de aplicaciones existentes en el mercado y en las fuentes bibliográficas, respecto al tema general de la investigación, además de la triangulación con las tres fuentes anteriormente nombradas, con el objetivo de conocer la evolución de dicho tema hasta la actualidad y proponer algunas características que pueden ser implementadas en los videojuegos gracias a la tecnología NFC.

Las secciones están divididas de la siguiente manera: 1. Metodología de evaluación, 2. Clasificación de videojuegos para dispositivos móviles, 3. Contexto comercial, 4. Estado actual en la tienda de aplicaciones, 5. Estado actual de la revisión literaria, 6. Síntesis de alternativas en videojuegos con NFC, 7. Conclusiones y 8. Agradecimientos.

\section{METODOLOGÍA DE EVALUACIÓN}

El enfoque de metodología de evaluación consiste en una triangulación de tres fuentes tal como muestra la figura 1; para ello se hace necesario establecer una clasificación de videojuegos la cual se encuentra en el apartado 2.

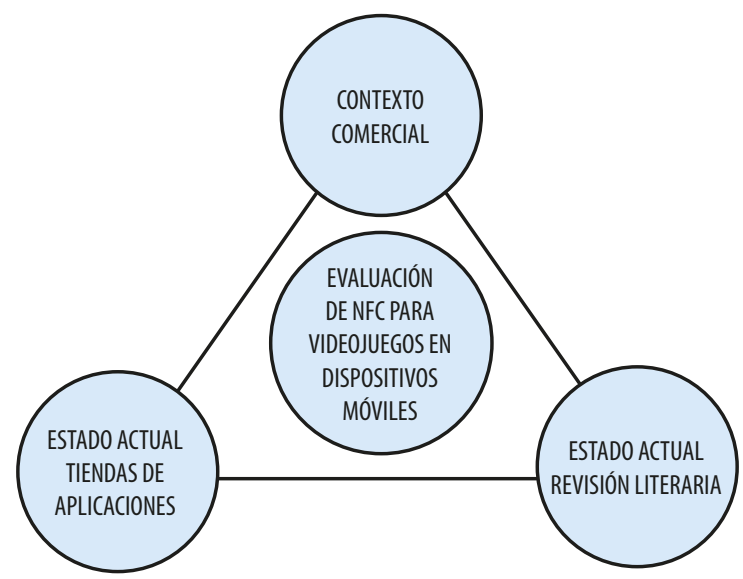

Figura 1. Metodología de evaluación: triangulación de fuentes de información.

Fuente: elaboración propia

Para determinar el estado actual y las futuras direcciones de la investigación en uso de la tecnología NFC para dispositivos móviles en videojuegos, se llevó a cabo una extensa revisión bibliográfica. 
Como primera medida se optó por determinar el alcance de la revisión de fuentes pertinentes. Se realizó una búsqueda en fuentes de información comercial, en las tiendas de aplicaciones existentes en el mercado para dispositivos móviles y en fuentes bibliográficas. Se tuvieron en cuenta los artículos y los proyectos que se han publicado sobre el tema en cuestión y los videojuegos existentes para dispositivos móviles que toman como base la tecnología NFC, los cuales se transformarán en insumos esenciales de esta investigación, ya que al ser estudiados a profundidad permitirán un mejor desarrollo de esta.

\section{CLASIFICACIÓN DE VIDEOJUEGOS PARA DISPOSITIVOS MÓVILES}

Para la clasificación de los videojuegos para dispositivos móviles se realizó una búsqueda de artículos en los cuales se encontraron los géneros de videojuegos, entre los cuales están las propuestas mencionadas en [1-13].

Una vez terminada dicha búsqueda se encontró que la clasificación de los videojuegos en los artículos no es siempre constante; tampoco se tiene una visión unificada de los autores y algunas veces puede ser algo arbitraria; por lo tanto, se optó por cruzar todos los géneros encontrados en dichos artículos, para así crear una lista que intente unificar las ideas de los autores y presente una categorización adecuada de los videojuegos. Los géneros clasificados se presentan en la tabla 1.

Tabla 1. Categorías de videojuegos propuestas

\begin{tabular}{|c|c|c|}
\hline Categoría & & Sección \\
\hline \multirow{5}{*}{ Acción } & \multicolumn{2}{|c|}{ Plataforma } \\
\hline & \multirow{3}{*}{ Disparo } & Disparos en primera persona \\
\hline & & Disparos en tercera persona \\
\hline & & Sigilo \\
\hline & \multicolumn{2}{|l|}{ Lucha } \\
\hline \multicolumn{3}{|l|}{ Aventura } \\
\hline \multicolumn{3}{|l|}{ Rol } \\
\hline \multirow{5}{*}{ Simulación } & \multicolumn{2}{|c|}{ Vehículos } \\
\hline & \multicolumn{2}{|c|}{ Construcción y gestión } \\
\hline & \multicolumn{2}{|c|}{ Deportes y participación } \\
\hline & \multicolumn{2}{|l|}{ Carreras } \\
\hline & \multicolumn{2}{|c|}{ Vida artificial } \\
\hline
\end{tabular}


NFC como alternativa para mejorar el desarrollo de videojuegos en dispositivos móviles 155

\begin{tabular}{|l|l|}
\hline \multicolumn{1}{|c|}{ Categoría } & \multicolumn{1}{c|}{ Sección } \\
\hline \multirow{2}{*}{ Estrategia } & Estrategia por turnos \\
\cline { 2 - 2 } & Estrategia en tiempo real \\
\hline Rompecabezas & \\
\hline Casuales o tradicionales & \\
\hline Multijugador masivo en línea & \\
\hline Música o ritmo & \\
\hline Educativos & \\
\hline Arcade & \\
\hline Búsqueda de tesoros & \\
\hline Realidad alternativa & \\
\hline Interpersonales & \\
\hline Laberinto & \\
\hline Basados en Ping-Pong & \\
\hline Captura & \\
\hline Recolección & \\
\hline Esquivando & \\
\hline Preguntas y respuestas & \\
\hline
\end{tabular}

Fuente: elaboración propia

La información presentada en la tabla 1 será utilizada posteriormente para clasificar los videojuegos analizados en la presente investigación.

\section{CONTEXTO COMERCIAL}

La fuente número uno para la investigación es el contexto comercial. En este apartado se realizó una búsqueda aleatoria en Internet de noticias referentes al tema de investigación, con el objetivo de tener una visión general sobre qué se está desarrollando o se ha desarrollado en el entorno respecto a la tecnología NFC y su empleo en videojuegos para dispositivos móviles; dicha búsqueda fue realizada mediante las palabras clave: "NFC", "NFC" AND "videogames", "NFC" AND "videojuegos", "NFC" AND "news", "NFC" AND "noticias", "NFC" AND "mobile devices" y "NFC" AND "dispositivos móviles".

En la tabla 2 se presentan algunas de las noticias encontradas entre septiembre y octubre de 2014; fueron seleccionadas las más representativas del tema en cuestión; 
además, se descartaron noticias cuya información ya se encontraba condensada en las presentadas en la tabla.

Tabla 2. Noticias encontradas más representativas del tema en cuestión

\begin{tabular}{|l|c|}
\hline \multicolumn{1}{|c|}{ Noticia } & Referencia \\
\hline Nintendo Amiibo, la Wii U usará figuras reales NFC en sus videojuegos & {$[14]$} \\
\hline ¿Qué dispositivos tienen NFC? & {$[15]$} \\
\hline Pronóstico: 400 \% incremento de ventas NFC en 2018 & {$[16]$} \\
\hline La tecnología NFC convierte las mesas de McDonald's en circuitos de carreras & {$[17]$} \\
\hline Angry Birds Magic traerá el NFC para los juegos & {$[18]$} \\
\hline Más muestras del potencial de iBeacon, videojuegos para iOS & {$[19]$} \\
\hline La tecnología iBeacon se usará en un juego promocional durante el CES & {$[20]$} \\
\hline iBeacon: The Underrated, Game-changing Technology of 2014 & {$[21]$} \\
\hline
\end{tabular}

Fuente: elaboración propia

Se destaca entre la búsqueda de noticias que la empresa Apple no implementa la tecnología NFC en sus dispositivos móviles, sino que ha desarrollado iBeacon como sustituto a ella; por esto se presentan también algunas de las noticas encontradas referentes a dicha tecnología.

Dado que los dispositivos de Apple proporcionan al usuario una tecnología equivalente a NFC, esta similitud podría despertar el interés de investigar sobre posibles usos en los videojuegos para dispositivos móviles, lo que haría parte de otra investigación para iBeacon.

A partir de la búsqueda de información comercial y noticias existentes en Internet se puede resaltar Angry Birds Magic como el videojuego con más difusión mediante mercadeo; esto debido a que en un gran número de las noticias encontradas se hablaba del lanzamiento de dicho videojuego; en una de ellas se mencionaba que las ventas globales de los dispositivos móviles con tecnología NFC se incrementarán en un $400 \%$ hasta 2018, alcanzando la cifra de 1200 millones de unidades.

\section{ESTADO ACTUAL EN TIENDAS DE APLICACIONES}

La fuente número dos es el estado actual en tiendas de aplicaciones existentes en el mercado. En este apartado se presenta la búsqueda realizada en las tiendas de aplicaciones para dispositivos móviles: Google Play Store, Nokia Store, BlackBerry World y Windows Phone, con el objetivo de encontrar los juegos que han sido desarrollados con la tecnología NFC y publicados en dichas tiendas; esta búsqueda fue realizada 
mediante las palabras clave: "NFC", "NFC" AND "videogames", "NFC" AND "videojuegos", "NFC" AND "games" y "NFC" AND "juegos".

Una vez encontrado cada juego, se organizó en la tabla 3, donde se resalta el nombre con el cual aparece en la tienda de aplicaciones, el sistema operativo en el que puede ser instalado, la función de la tecnología NFC en dicho juego y la categoría a la que pertenece.

En dicha tabla se presentan los diecisiete (17) videojuegos que utilizan NFC y han sido publicados en tiendas de aplicaciones para dispositivos móviles; además, se hace un análisis de la información que esta presenta de la siguiente manera.

- Sistema operativo

Ciertos videojuegos se encuentran disponibles para sistemas operativos que están siendo menos utilizados e implementados en los dispositivos móviles actuales como

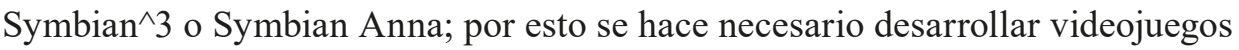
con NFC en los sistemas operativos más usados en la actualidad.

- Uso de NFC

Los usos más comunes en los videojuegos con NFC de las tiendas de aplicaciones son los de aproximación entre dispositivos móviles o tarjetas de esta misma tecnología para desbloquear nuevo contenido para los juegos (siendo este modo utilizado en diez de ellos), observar el juego en ambas pantallas de los dispositivos móviles utilizados, acercar el dispositivo a las etiquetas NFC que representen el contenido que aparece en el juego, retar a un compañero en modo multi-jugador y utilizar cada etiqueta NFC como un punto de control.

- Categoría

Las categorías que sobresalen en la tabla 3 de acuerdo con el número de videojuegos, en orden descendente son: educativo, arcade, interpersonales y lucha.

Tabla 3. Videojuegos con NFC publicados en las tiendas de aplicaciones

\begin{tabular}{|l|l|l|l|}
\hline \multicolumn{1}{|c|}{ Nombre } & \multicolumn{1}{|c|}{ Sistema Operativo } & \multicolumn{1}{c|}{ ¿Qué hace NFC? } & \multicolumn{1}{c|}{ Categoría } \\
\hline Angry Birds Magic & Nokia Belle & $\begin{array}{l}\text { Desbloquear nuevos niveles } \\
\text { Obtener recompensas exclusivas } \\
\text { Conseguir productos exclusivos }\end{array}$ & $\begin{array}{l}\text { Estrategia por turnos, } \\
\text { arcade }\end{array}$ \\
\hline Fruit Ninja & Symbian^3 & Desbloquear nuevo contenido & $\begin{array}{l}\text { Estrategia en tiempo } \\
\text { real, arcade }\end{array}$ \\
\hline NFC-Bots & $\begin{array}{l}\text { Android 2.0 o } \\
\text { superior }\end{array}$ & $\begin{array}{l}\text { Obtener nuevas piezas en el juego } \\
\text { para el robot acercando el dispositi- } \\
\text { vo a una pieza real }\end{array}$ & Lucha \\
\hline
\end{tabular}


Stiven Muñoz-Quintero - Juan Rincón Patino - Gustavo Ramirez-Gonzalez

\begin{tabular}{|c|c|c|c|}
\hline Nombre & Sistema Operativo & ¿Qué hace NFC? & Categoría \\
\hline NFC Hunter & $\begin{array}{l}\text { Android } 2.3 .30 \\
\text { superior }\end{array}$ & $\begin{array}{l}\text { Al acercar el dispositivo a una } \\
\text { etiqueta NFC aparecerá el monstruo } \\
\text { que se debe golpear }\end{array}$ & Lucha \\
\hline Near Field Ninja & $\begin{array}{l}\text { Android } 4.0 \mathrm{o} \\
\text { superior }\end{array}$ & $\begin{array}{l}\text { El juego se observa en las dos } \\
\text { pantallas de los jugadores por medio } \\
\text { de NFC }\end{array}$ & $\begin{array}{l}\text { Estrategia por turnos, } \\
\text { acción, interpersonal }\end{array}$ \\
\hline Nokia World Flags & $\begin{array}{l}\text { Symbian Anna, } \\
\text { Nokia Belle }\end{array}$ & $\begin{array}{l}\text { Se acerca el teléfono a tarjetas NFC } \\
\text { para revelar banderas ocultas }\end{array}$ & Educativo \\
\hline $\begin{array}{l}\text { Nokia Nursery } \\
\text { Rhyme Shuffle }\end{array}$ & $\begin{array}{l}\text { Symbian Anna, } \\
\text { Nokia Belle }\end{array}$ & $\begin{array}{l}\text { Al pasar el dispositivo cerca de una } \\
\text { tarjeta NFC se escuchará una parte } \\
\text { de una cita de obras de los } 90\end{array}$ & $\begin{array}{l}\text { Rompecabezas, musi- } \\
\text { cal, educativo }\end{array}$ \\
\hline $\begin{array}{l}\text { Nokia Shakespeare } \\
\text { Shuffle }\end{array}$ & $\begin{array}{l}\text { Symbian Anna, } \\
\text { Nokia Belle }\end{array}$ & $\begin{array}{l}\text { Al pasar el dispositivo cerca de una } \\
\text { tarjeta NFC se escuchará una parte } \\
\text { de una cita de Shakespeare }\end{array}$ & $\begin{array}{l}\text { Rompecabezas, musi- } \\
\text { cal, educativo }\end{array}$ \\
\hline Revolver: NFC App & $\begin{array}{l}\text { Symbian Anna, } \\
\text { Nokia Belle }\end{array}$ & $\begin{array}{l}\text { Al acercar a un dispositivo con } \\
\text { NFC o una tarjeta NFC se dispara } \\
\text { el revólver }\end{array}$ & Simulación \\
\hline $\begin{array}{l}\text { Bounce Boing } \\
\text { Battle }\end{array}$ & $\begin{array}{l}\text { Symbian^3, Sym- } \\
\text { bian Anna, Nokia } \\
\text { Belle }\end{array}$ & $\begin{array}{l}\text { Al acercar a un dispositivo con NFC } \\
\text { el juego se verá en la pantalla de } \\
\text { los dos dispositivos (la mitad de la } \\
\text { pantalla en cada uno) }\end{array}$ & Interpersonal, arcade \\
\hline NFC Shapes & Symbian^3 & $\begin{array}{l}\text { Deben coincidir las formas que apa- } \\
\text { recen en el teléfono con la etiqueta } \\
\text { NFC correcta }\end{array}$ & Educativo \\
\hline NFC Colours & Symbian^3 & $\begin{array}{l}\text { Deben coincidir los colores que apa- } \\
\text { recen en el teléfono con la etiqueta } \\
\text { NFC correcta }\end{array}$ & Educativo \\
\hline Camagotchi & Windows Phone 8 & $\begin{array}{l}\mathrm{Al} \text { acercar a un dispositivo con NFC } \\
\text { que tenga el juego, los camellos de } \\
\text { los dos dispositivos son alimentados }\end{array}$ & Interpersonal, arcade \\
\hline Smash Mania & Nokia Belle & $\begin{array}{l}\text { Con NFC se puede retar a un com- } \\
\text { pañero en modo multi-jugador }\end{array}$ & $\begin{array}{l}\text { Deporte, } \\
\text { interpersonal }\end{array}$ \\
\hline NFC Battler & $\begin{array}{l}\text { Android } 2.30 \\
\text { superior }\end{array}$ & $\begin{array}{l}\text { Desbloquear máscaras para el } \\
\text { luchador }\end{array}$ & Lucha \\
\hline Treasure Hunt & $\begin{array}{l}\text { Android } 2.3 .3 \text { o } \\
\text { superior, Windows } \\
\text { Phone } 8\end{array}$ & $\begin{array}{l}\text { Cada punto de control es una tarjeta } \\
\text { NFC, se permite compartir caminos } \\
\text { ya realizados y los mejores tiempos. }\end{array}$ & $\begin{array}{l}\text { Búsqueda de tesoros, } \\
\text { multijugador }\end{array}$ \\
\hline $\begin{array}{l}\text { NFC Rock Paper } \\
\text { Scissors }\end{array}$ & $\begin{array}{l}\text { BlackBerry } 10 \text { OS } \\
\text { Windows Phone } 8\end{array}$ & $\begin{array}{l}\text { Juego de piedra, papel o tijera, } \\
\text { utilizando dos dispositivos móviles } \\
\text { con NFC }\end{array}$ & Interpersonal, casual \\
\hline
\end{tabular}


Una versión ampliada de la tabla 3 se puede encontrar en [22]. De la tabla 3 se puede resaltar que cinco (5) de los juegos pueden ser instalados en dispositivos Android y doce (12) en dispositivos Nokia; esto se debe a que la gran mayoría de los juegos han sido desarrollados años atrás, tiempo en que Nokia era la empresa que más había implementado NFC en sus móviles. Solo uno de los juegos puede ser instalado en dispositivos móviles BlackBerry. Se debe tener en cuenta que la búsqueda se realizó entre los meses de septiembre a diciembre de 2014; por lo tanto, los juegos que estaban disponibles en la tienda Nokia Store ahora no lo están, ya que dicha tienda ha sido sustituida actualmente por Opera Mobile Store como la tienda de aplicaciones para teléfonos de las familias Series 40, Series 60, Symbian, Asha y Nokia X [23], eliminando así muchos juegos de la tienda anterior.

\section{ESTADO ACTUAL DE LA REVISIÓN LITERARIA}

Como última fuente se cuenta con el estado actual de la revisión literaria, para lo cual se realizó una búsqueda con una amplia exploración sistemática de las bases de datos de revistas y conferencias académicas en línea, entre las cuales fueron seleccionadas: ScienceDirect, IEEE Xplore, Springer, ACM Digital Library y Google Scholar; de los documentos identificados también se hizo una búsqueda mediante la revisión de otros trabajos de los mismos autores; además, se inspeccionó cada uno de los documentos que citan los escritos anteriores.

Para la búsqueda se definió una serie de palabras clave en español e inglés, las cuales se debían encontrar en el título o el resumen de las fuentes anteriormente nombradas; estas permitieron filtrar de una manera efectiva toda la información disponible; se partió desde el término general "NFC", luego se realizó una combinación de las palabras clave que permitió encontrar información importante para el tema de interés; dichas palabras se observan en la tabla 4; cabe resaltar que la palabra clave "videojuegos" no entrega un número alto de resultados relevantes para la investigación; además, la mayoría de la información literaria es encontrada en inglés, lo que hace que dicha palabra no fuese utilizada en muchas de las combinaciones.

Tabla 4. Palabras clave utilizadas para la revisión literaria

\begin{tabular}{|l|l|}
\hline \multicolumn{2}{|c|}{ Palabras clave } \\
\hline A & "Near Field Communication" \\
\hline B & "Near Field Communication" AND "videojuegos" \\
\hline C & "Near Field Communication" AND "videogames" \\
\hline D & "Near Field Communication" AND "games" \\
\hline
\end{tabular}




\begin{tabular}{|c|c|}
\hline \multicolumn{2}{|r|}{ Palabras clave } \\
\hline $\mathrm{E}$ & "Near Field Communication" AND "smartphones" \\
\hline $\mathrm{F}$ & "Near Field Communication” AND “dispositivos móviles” \\
\hline G & "Near Field Communication" AND "mobile devices" \\
\hline $\mathrm{H}$ & "Near Field Communication" AND “videojuegos" AND “dispositivos móviles" \\
\hline I & "Near Field Communication" AND "videogames" AND “smartphones" \\
\hline $\mathrm{J}$ & "Near Field Communication" AND “videogames" AND "mobile devices" \\
\hline $\mathrm{K}$ & "Near Field Communication" AND "videogames" AND "Android" \\
\hline $\mathrm{L}$ & "Near Field Communication” AND “videogames” AND “Nokia” \\
\hline M & "Near Field Communication" AND "games" AND "smartphones" \\
\hline $\mathrm{N}$ & "Near Field Communication” AND “games" AND "mobile devices" \\
\hline $\mathrm{O}$ & "Near Field Communication" AND "games" AND “Android" \\
\hline $\mathrm{P}$ & "Near Field Communication" AND “games" AND “Nokia” \\
\hline
\end{tabular}

Fuente: elaboración propia

Al realizar la búsqueda para cada combinación de la tabla 4 se obtiene el número de artículos que se presenta en la tabla 5; dicho número es el total de documentos que arroja cada fuente bibliográfica sin ser todavía filtrados para encontrar los referentes de interés de la presente investigación; se hace importante explicar que en la tabla 5 no se presenta el cálculo total que sí se registra en las tablas siguientes; además, el número de resultados arrojados por Google Scholar es significativamente mayor. Estas dos aclaraciones suceden, ya que dicha fuente bibliográfica indexa los resultados de las demás lo cual genera que muchos de los artículos encontrados en estas últimas ya se encuentren en los resultados obtenidos en Google Scholar, pero esto no disminuye la importancia de la búsqueda en ellas, ya que son necesarias para afinar la investigación y obtener resultados de búsqueda que en el caso genérico no están entre los primeros.

Tabla 5. Resultado búsqueda palabras clave

\begin{tabular}{|c|c|c|c|c|c|}
\hline $\begin{array}{c}\text { Palabras clave en la } \\
\text { búsqueda }\end{array}$ & $\begin{array}{c}\text { Science } \\
\text { Direct }\end{array}$ & $\begin{array}{c}\text { IEEE } \\
\text { Xplore }\end{array}$ & Springer & ACM & $\begin{array}{c}\text { Google } \\
\text { Scholar }\end{array}$ \\
\hline A & 439 & 2660 & 941 & 913 & 25700 \\
\hline B & 0 & 0 & 1 & 0 & 29 \\
\hline C & 1 & 1 & 1 & 3 & 40 \\
\hline D & 104 & 21 & 237 & 149 & 4120 \\
\hline E & 97 & 37 & 255 & 196 & 4970 \\
\hline
\end{tabular}


NFC como alternativa para mejorar el desarrollo de videojuegos en dispositivos móviles 161

\begin{tabular}{|c|c|c|c|c|c|}
\hline $\begin{array}{c}\text { Palabras clave en la } \\
\text { búsqueda }\end{array}$ & $\begin{array}{c}\text { Science } \\
\text { Direct }\end{array}$ & $\begin{array}{c}\text { IEEE } \\
\text { Xplore }\end{array}$ & Springer & ACM & $\begin{array}{c}\text { Google } \\
\text { Scholar }\end{array}$ \\
\hline F & 0 & 0 & 1 & 0 & 180 \\
\hline G & 195 & 214 & 418 & 439 & 9020 \\
\hline H & 0 & 0 & 1 & 0 & 20 \\
\hline I & 1 & 0 & 1 & 2 & 21 \\
\hline J & 1 & 1 & 1 & 2 & 26 \\
\hline K & 1 & 0 & 1 & 0 & 23 \\
\hline L & 0 & 0 & 0 & 1 & 15 \\
\hline M & 36 & 2 & 68 & 61 & 1230 \\
\hline N & 62 & 4 & 148 & 109 & 2320 \\
\hline O & 19 & 0 & 78 & 54 & 1090 \\
\hline P & 28 & 0 & 69 & 58 & 878 \\
\hline
\end{tabular}

Fuente: elaboración propia

Posteriormente, se comenzó a revisar cada uno de los artículos encontrados por su título, observando que guardase relación con el tema de investigación; cabe resaltar que al avanzar en la búsqueda los resultados pierden relevancia; por tanto, cuando no se encuentran resultados importantes durante un período prolongado se opta por terminar la revisión de la palabra clave en cuestión. En caso que el título guardase relación con la presente investigación se observó el resumen y los autores de dicho artículo para determinar si puede ser uno de los referentes. El número de artículos revisados en su totalidad se presenta en la tabla 6 .

Tabla 6. Artículos revisados por título, resumen y autores

\begin{tabular}{|c|c|c|c|c|c|c|}
\hline $\begin{array}{c}\text { Palabras clave en la } \\
\text { búsqueda }\end{array}$ & $\begin{array}{c}\text { Science } \\
\text { Direct }\end{array}$ & $\begin{array}{c}\text { IEEE } \\
\text { Xplore }\end{array}$ & Springer & ACM & $\begin{array}{c}\text { Google } \\
\text { Scholar }\end{array}$ & Total \\
\hline A & 30 & 59 & 36 & 34 & 68 & 227 \\
\hline B & 0 & 0 & 0 & 0 & 2 & 2 \\
\hline C & 0 & 1 & 0 & 3 & 6 & 10 \\
\hline D & 18 & 5 & 7 & 36 & 14 & 80 \\
\hline E & 12 & 7 & 3 & 24 & 12 & 58 \\
\hline F & 0 & 0 & 0 & 0 & 4 & 4 \\
\hline G & 7 & 13 & 9 & 21 & 5 & 55 \\
\hline
\end{tabular}


Stiven Muñoz-Quintero - Juan Rincón Patino - Gustavo Ramirez-Gonzalez

\begin{tabular}{|c|c|c|c|c|c|c|}
\hline $\begin{array}{c}\text { Palabras clave en la } \\
\text { búsqueda }\end{array}$ & $\begin{array}{c}\text { Science } \\
\text { Direct }\end{array}$ & $\begin{array}{c}\text { IEEE } \\
\text { Xplore }\end{array}$ & Springer & ACM & $\begin{array}{c}\text { Google } \\
\text { Scholar }\end{array}$ & Total \\
\hline H & 0 & 0 & 0 & 0 & 1 & 1 \\
\hline I & 1 & 0 & 0 & 2 & 0 & 3 \\
\hline J & 1 & 0 & 0 & 2 & 5 & 8 \\
\hline K & 1 & 0 & 0 & 0 & 3 & 4 \\
\hline L & 0 & 0 & 0 & 1 & 3 & 4 \\
\hline M & 2 & 2 & 4 & 11 & 2 & 21 \\
\hline N & 10 & 0 & 3 & 14 & 1 & 28 \\
\hline O & 2 & 0 & 1 & 4 & 1 & 8 \\
\hline P & 1 & 0 & 0 & 3 & 2 & 6 \\
\hline TOTAL & 85 & 87 & 63 & 155 & 129 & 519 \\
\hline
\end{tabular}

Fuente: elaboración propia

Enseguida se procede a seleccionar aquellos artículos que sean relevantes para la investigación y se ordenan según la fuente bibliográfica en la cual fueron encontrados; eso se presenta en la tabla 7.

Tabla 7. Referentes encontrados de interés para la investigación

\begin{tabular}{|c|c|c|c|c|c|c|}
\hline $\begin{array}{c}\text { Palabras clave en la } \\
\text { búsqueda }\end{array}$ & $\begin{array}{c}\text { Science } \\
\text { Direct }\end{array}$ & $\begin{array}{c}\text { IEEE } \\
\text { Xplore }\end{array}$ & Springer & ACM & $\begin{array}{c}\text { Google } \\
\text { Scholar }\end{array}$ & Total \\
\hline A & 3 & 12 & 3 & 1 & 4 & 23 \\
\hline B & 0 & 0 & 0 & 0 & 0 & 0 \\
\hline C & 0 & 1 & 0 & 0 & 3 & 4 \\
\hline D & 2 & 0 & 0 & 2 & 4 & 8 \\
\hline E & 3 & 0 & 0 & 0 & 1 & 4 \\
\hline F & 0 & 0 & 0 & 0 & 0 & 0 \\
\hline G & 0 & 2 & 0 & 1 & 0 & 3 \\
\hline H & 0 & 0 & 0 & 0 & 0 & 0 \\
\hline I & 0 & 0 & 0 & 0 & 0 & 0 \\
\hline J & 0 & 0 & 0 & 0 & 0 & 0 \\
\hline K & 0 & 0 & 0 & 0 & 0 & 0 \\
\hline L & 0 & 0 & 0 & 0 & 0 & 0 \\
\hline M & 0 & 0 & 1 & 1 & 0 & 2 \\
\hline
\end{tabular}


NFC como alternativa para mejorar el desarrollo de videojuegos en dispositivos móviles 163

\begin{tabular}{|c|c|c|c|c|c|c|}
\hline $\begin{array}{c}\text { Palabras clave en la } \\
\text { búsqueda }\end{array}$ & $\begin{array}{c}\text { Science } \\
\text { Direct }\end{array}$ & $\begin{array}{c}\text { IEEE } \\
\text { Xplore }\end{array}$ & Springer & ACM & $\begin{array}{c}\text { Google } \\
\text { Scholar }\end{array}$ & Total \\
\hline N & 0 & 0 & 0 & 0 & 0 & 0 \\
\hline O & 0 & 0 & 0 & 0 & 0 & 0 \\
\hline P & 0 & 0 & 0 & 1 & 0 & 1 \\
\hline TOTAL & 8 & 15 & 4 & 6 & 12 & 45 \\
\hline
\end{tabular}

Fuente: elaboración propia

Luego, se ordenó cada uno de los referentes que fueron seleccionados, de acuerdo con cada fuente bibliográfica; para ello se tiene en cuenta: el título, un resumen, la fecha de publicación, el autor o los autores, la organización en que fue desarrollado y las ideas principales de cada uno de ellos, permitiendo así destacar que en los referentes de interés para la investigación se trabajan criterios relevantes como:

- Presentar el diseño, implementación o evaluación de un videojuego que haga uso de la tecnología NFC.

- Mostrar alguna aplicación de NFC, sin ser necesariamente un videojuego, con el objetivo de conocer los diferentes usos de esta tecnología.

- Describir las propiedades de algunos videojuegos ubicuos que puedan ser implementadas con NFC.

- Explicar algunas ventajas y desventajas de la tecnología en cuestión.

De cada uno de los referentes de la investigación se observó el año en que fue publicado para así saber cómo ha sido la evolución o el uso de NFC con videojuegos, lo cual se observa en la figura 2 .

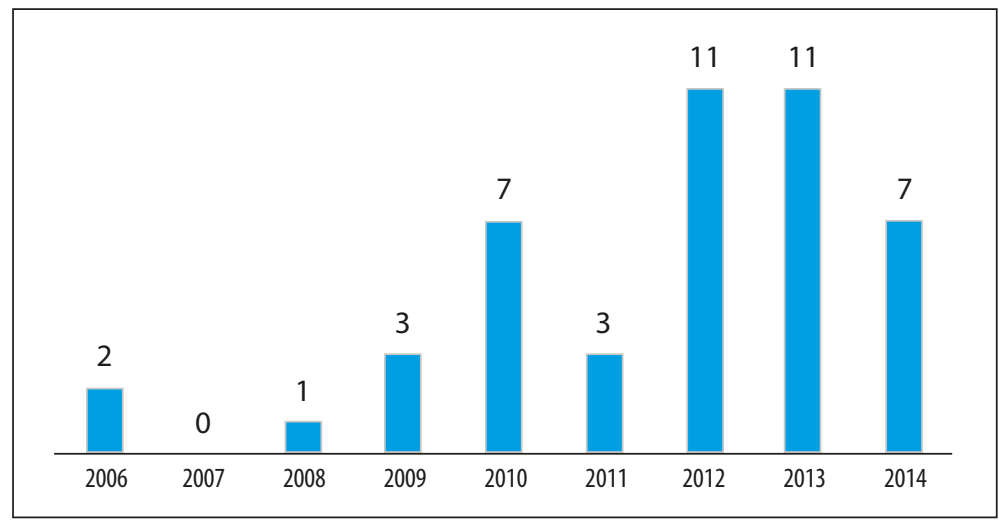

Figura 2. Número de referentes de la investigación publicados por año. 
Una versión ampliada de la figura 2 se puede encontrar en [22]. Dicha figura permite inferir que el desarrollo de NFC para videojuegos en dispositivos móviles ha estado en crecimiento, puesto que el número de artículos publicados muestra una tendencia en aumento en los últimos años; se resalta que el resultado del 2014 corresponde a la búsqueda realizada a septiembre de ese mismo año.

De los referentes de interés, también se extrajeron los videojuegos con NFC para dispositivos móviles que se diseñaban, implementaban, evaluaban o mencionaban en cada uno de los 45 referentes de la investigación; dichos videojuegos son presentados en la tabla 8. Al haber realizado dicha extracción, se hizo el siguiente análisis por columnas de la tabla:

- Categoría

Las categorías que sobresalen de la tabla 8 , de acuerdo con el número de videojuegos en orden descendente, son: estrategia, casual, interpersonales, educativo, rompecabezas y búsqueda de tesoros; esto, debido a que NFC permite implementar videojuegos en estas categorías, que mejoran la interacción presencial de los usuarios con objetos o entre ellos mismos, haciendo uso de las etiquetas y otras de sus propiedades.

- Sistema operativo

Muchos de los videojuegos han sido desarrollados para sistemas operativos que están siendo menos utilizados e implementados en los dispositivos móviles actuales como Nokia OS Series 40 o Nokia Belle; esto hace necesario que los desarrollos de nuevos videojuegos con NFC sea en los sistemas operativos posicionados en la actualidad, con el objetivo de explotar mayores propiedades de dichos sistemas operativos y poder ser jugados en un mayor número de dispositivos.

- Uso de NFC

En los videojuegos mencionados en los 45 referentes de interés de la investigación, también se tienen como usos más comunes los mencionados en el apartado 4, donde se encuentran los videojuegos disponibles en las tiendas de aplicaciones para dispositivos móviles.

Es importante destacar que muchos de los videojuegos mencionados en la tabla 8 hacen uso de etiquetas NFC y RFID, mientras que unos pocos se complementan con otras tecnologías tales como Wi-Fi (The Transhumance Project y NFC Heroes), Bluetooth (Whack-a-Mole) o GPS (PACLAN, Seek-It \& Touch-It, Mobspray y MobHunt), siendo una buena opción la de combinar diferentes tecnologías junto con NFC para mejorar la experiencia del usuario y la usabilidad del videojuego. 
Tabla 8. Videojuegos encontrados en los referentes de interés de la investigación

\begin{tabular}{|c|c|c|c|c|}
\hline Nombre & Categoría & Sistema Operativo & ¿Qué hace NFC? & Referencia \\
\hline CachedSensing & Búsqueda de tesoros & Android & $\begin{array}{l}\text { Se permite leer etiquetas NFC } \\
\text { que contienen información } \\
\text { sobre el sitio o el artículo } \\
\text { encontrado }\end{array}$ & {$[24]$} \\
\hline PACLAN & $\begin{array}{l}\text { Arcade, rompecabezas, } \\
\text { estrategia en tiempo } \\
\text { real }\end{array}$ & $\begin{array}{l}\text { Nokia OS Series } \\
40 \text { 3rd Edition }\end{array}$ & $\begin{array}{l}\text { Los jugadores deben recoger } \\
\text { píldoras que son etiquetas NFC }\end{array}$ & $\begin{array}{c}{[25-26]} \\
{[2]}\end{array}$ \\
\hline Hot in the city & Interpersonales & $\begin{array}{l}\text { Desconocido } \\
\text { (móviles con } \\
\text { NFC) }\end{array}$ & $\begin{array}{l}\text { La información de las personas } \\
\text { puede ser grabada en etiquetas } \\
\text { NFC. }\end{array}$ & {$[27,28]$} \\
\hline Pass the bomb & $\begin{array}{l}\text { Interpersonales, estra- } \\
\text { tegia por turnos }\end{array}$ & $\begin{array}{l}\text { Nokia OS Series } \\
40 \text { 3rd Edition }\end{array}$ & $\begin{array}{l}\text { Pasa la bomba de la pantalla } \\
\text { de uno de los jugadores a la } \\
\text { pantalla del siguiente }\end{array}$ & [29] \\
\hline $\begin{array}{l}\text { Exquisite } \\
\text { touch }\end{array}$ & $\begin{array}{l}\text { Interpersonales, } \\
\text { música }\end{array}$ & $\begin{array}{l}\text { Nokia OS Series } \\
40 \text { 3rd Edition }\end{array}$ & $\begin{array}{l}\text { Pasa los datos de las preguntas } \\
\text { realizadas al celular del siguien- } \\
\text { te turno }\end{array}$ & [29] \\
\hline $\begin{array}{l}\text { Seek-It \& } \\
\text { Touch-It }\end{array}$ & $\begin{array}{l}\text { Búsqueda de tesoros, } \\
\text { estrategia en tiempo } \\
\text { real }\end{array}$ & $\begin{array}{l}\text { Nokia OS Series } \\
40 \text { 3rd Edition }\end{array}$ & $\begin{array}{l}\text { Presenta la información del ob- } \\
\text { jeto encontrado y lo dirige a la } \\
\text { plataforma Moodle }\end{array}$ & [29] \\
\hline Whack-a-Mole & Casual & $\begin{array}{l}\text { Nokia OS Series } \\
40 \text { 5th Edition }\end{array}$ & $\begin{array}{l}\text { Se ponen etiquetas NFC tras } \\
\text { de una cortina que simula una } \\
\text { pantalla dinámica, así se detec- } \\
\text { ta dónde golpea el jugador }\end{array}$ & {$[30,31]$} \\
\hline $\begin{array}{l}\text { Excavate and } \\
\text { learn }\end{array}$ & Simulación & $\begin{array}{l}\text { Desconocido } \\
\text { (móviles con } \\
\text { NFC) }\end{array}$ & $\begin{array}{l}\text { Presenta la información del ob- } \\
\text { jeto al cual se acerca el visitante }\end{array}$ & {$[32]$} \\
\hline $\begin{array}{l}\text { NFC Mat- } \\
\text { ching Game }\end{array}$ & $\begin{array}{l}\text { Casual, estrategia por } \\
\text { turnos }\end{array}$ & $\begin{array}{l}\text { Symbian Anna, } \\
\text { Nokia Belle }\end{array}$ & $\begin{array}{l}\text { Cada etiqueta representa una } \\
\text { imagen, el jugador puede desta- } \\
\text { par dos de ellas por turno }\end{array}$ & {$[33]$} \\
\hline $\begin{array}{l}\text { NFC Drum } \\
\text { Repeat }\end{array}$ & $\begin{array}{l}\text { Casual, estrategia por } \\
\text { turnos }\end{array}$ & $\begin{array}{l}\text { Symbian Anna, } \\
\text { Nokia Belle }\end{array}$ & $\begin{array}{l}\text { Cada etiqueta NFC activa el } \\
\text { sonido de unos tambores dife- } \\
\text { rentes en el dispositivo }\end{array}$ & {$[33]$} \\
\hline $\begin{array}{l}\text { NFC Word } \\
\text { Shuffle }\end{array}$ & $\begin{array}{l}\text { Casual, estrategia por } \\
\text { turnos }\end{array}$ & $\begin{array}{l}\text { Symbian Anna, } \\
\text { Nokia Belle }\end{array}$ & $\begin{array}{l}\text { Las etiquetas NFC representan } \\
\text { una letra del alfabeto }\end{array}$ & {$[33]$} \\
\hline $\begin{array}{l}\text { NFC Gem } \\
\text { Shuffle }\end{array}$ & $\begin{array}{l}\text { Casual, estrategia por } \\
\text { turnos }\end{array}$ & $\begin{array}{l}\text { Symbian Anna, } \\
\text { Nokia Belle }\end{array}$ & $\begin{array}{l}\text { Descubre la gema que hay tras } \\
\text { de cada etiqueta NFC }\end{array}$ & [33] \\
\hline $\begin{array}{l}\text { NFC Shakes- } \\
\text { peare Shuffle }\end{array}$ & $\begin{array}{l}\text { Rompecabezas, música } \\
\text { y educativo }\end{array}$ & $\begin{array}{l}\text { Symbian Anna, } \\
\text { Nokia Belle }\end{array}$ & $\begin{array}{l}\text { Cada etiqueta NFC descubre } \\
\text { una cita de Shakespeare. }\end{array}$ & [33] \\
\hline
\end{tabular}




\begin{tabular}{|c|c|c|c|c|}
\hline Nombre & Categoría & Sistema Operativo & ¿Qué hace NFC? & Referencia \\
\hline $\begin{array}{l}\text { Nursery Rhy- } \\
\text { me Shuffle }\end{array}$ & $\begin{array}{l}\text { Rompecabezas, música } \\
\text { y educativo }\end{array}$ & $\begin{array}{l}\text { Symbian Anna, } \\
\text { Nokia Belle }\end{array}$ & $\begin{array}{l}\text { Al pasar el dispositivo cerca de } \\
\text { una tarjeta NFC se escuchará } \\
\text { una parte de una cita de obras } \\
\text { de los } 90 \text { 's. }\end{array}$ & [33] \\
\hline $\begin{array}{l}\text { The Snow } \\
\text { Game }\end{array}$ & Estrategia por turnos, & $\begin{array}{l}\text { Desconocido } \\
\text { (móviles con } \\
\text { NFC) }\end{array}$ & $\begin{array}{l}\text { Al tocar el dispositivo mó- } \\
\text { vil del otro jugador, se inter- } \\
\text { cambia la información de los } \\
\text { movimientos. }\end{array}$ & {$[34]$} \\
\hline $\begin{array}{l}\text { The Veggie } \\
\text { Game }\end{array}$ & $\begin{array}{l}\text { Estrategia en tiem- } \\
\text { po real, simulación, } \\
\text { interpersonales }\end{array}$ & $\begin{array}{l}\text { Desconocido } \\
\text { (móviles con } \\
\text { NFC) }\end{array}$ & $\begin{array}{l}\text { Al tocar los dispositivos con } \\
\text { NFC, se pueden intercambiar } \\
\text { vegetales y hacer crecer más } \\
\text { rápidamente otros. }\end{array}$ & {$[34]$} \\
\hline CountMeIn & $\begin{array}{l}\text { Estrategia por turnos, } \\
\text { rompecabezas }\end{array}$ & Android & $\begin{array}{l}\text { Pueden ayudar a completar la } \\
\text { secuencia varias personas que } \\
\text { tengan un dispositivo móvil } \\
\text { NFC }\end{array}$ & {$[35-36]$} \\
\hline $\begin{array}{l}\text { The Transhu- } \\
\text { mance project }\end{array}$ & $\begin{array}{l}\text { Búsqueda de tesoros, } \\
\text { interpersonales }\end{array}$ & $\begin{array}{l}\text { Desconocido } \\
\text { (móviles con } \\
\text { NFC) }\end{array}$ & $\begin{array}{l}\text { En cada lugar importante se } \\
\text { pone una etiqueta NFC que } \\
\text { contiene una pista para encon- } \\
\text { trar el sitio principal }\end{array}$ & [37] \\
\hline $\begin{array}{l}\text { Secrets of the } \\
\text { museum }\end{array}$ & Búsqueda de tesoros & $\begin{array}{l}\text { Nokia OS Series } \\
40 \text { 3rd Edition }\end{array}$ & $\begin{array}{l}\text { El jugador debe buscar etique- } \\
\text { tas de un mismo grupo para } \\
\text { ganar puntos }\end{array}$ & {$[37,38]$} \\
\hline TrainInAb & $\begin{array}{l}\text { Educativo, estrategia } \\
\text { por turnos }\end{array}$ & $\begin{array}{l}\text { Desconocido } \\
\text { (móviles con } \\
\text { NFC) }\end{array}$ & $\begin{array}{l}\text { Los objetos están identificados } \\
\text { con etiquetas NFC }\end{array}$ & [39] \\
\hline StiCap & $\begin{array}{l}\text { Educativo, estrategia } \\
\text { por turnos }\end{array}$ & $\begin{array}{l}\text { Desconocido } \\
\text { (móviles con } \\
\text { NFC) }\end{array}$ & $\begin{array}{l}\text { Los objetos están identificados } \\
\text { con etiquetas NFC }\end{array}$ & [39] \\
\hline $\begin{array}{l}\text { The Literature } \\
\text { Race }\end{array}$ & $\begin{array}{l}\text { Educativo, interperso- } \\
\text { nales, rompecabezas }\end{array}$ & Android & $\begin{array}{l}\text { Los libros se identifican con una } \\
\text { etiqueta NFC }\end{array}$ & {$[40]$} \\
\hline Mobspray & Simulación & $\begin{array}{l}\text { Nokia OS Series } \\
40 \text { 3rd Edition }\end{array}$ & $\begin{array}{l}\text { Los usuarios deben adquirir } \\
\text { unas tarjetas NFC que le per- } \\
\text { miten el ingreso al portal web }\end{array}$ & [41] \\
\hline MobHunt & Búsqueda de tesoros & $\begin{array}{l}\text { Nokia OS Series } \\
40 \text { 3rd Edition }\end{array}$ & $\begin{array}{l}\text { Las etiquetas contienen las pis- } \\
\text { tas para continuar en el camino } \\
\text { de la búsqueda }\end{array}$ & [41] \\
\hline NFC Heroes & $\begin{array}{l}\text { Casual, lucha, estrate- } \\
\text { gia por turnos, arcade, } \\
\text { multijugador en línea }\end{array}$ & $\begin{array}{l}\text { Android } 2.3 \mathrm{o} \\
\text { mayor }\end{array}$ & $\begin{array}{l}\text { Al acercarlos a unas etiquetas } \\
\text { NFC especiales, se desbloquean } \\
\text { poderes y héroes raros }\end{array}$ & {$[42,43]$} \\
\hline
\end{tabular}




\section{SÍNTESIS DE ALTERNATIVAS EN VIDEOJUEGOS CON NFC}

Al haber observado los resultados obtenidos en las tres fuentes de información es posible proponer algunas características que pueden ser implementadas en los futuros videojuegos desarrollados por generadores de contenido y emprendedores en dicho campo; estas características se encuentran condensadas en la tabla 9.

Tabla 9. Características que pueden ser implementadas con NFC

\begin{tabular}{|l|l|l|}
\hline \multirow{2}{*}{ Funcionalidad NFC } & $\begin{array}{l}\text { Característica en el } \\
\text { videojuego }\end{array}$ & \multicolumn{1}{|c|}{ Descripción característica } \\
\hline \multirow{2}{*}{$\begin{array}{l}\text { Leer etiquetas NFC } \\
\text { con el dispositivo } \\
\text { móvil }\end{array}$} & Cambio de & $\begin{array}{l}\text { De acuerdo a la etiqueta acercada al dispositivo móvil se } \\
\text { cambia el personaje del videojuego }\end{array}$ \\
\cline { 2 - 3 } & $\begin{array}{l}\text { Aumento de } \\
\text { habilidades }\end{array}$ & $\begin{array}{l}\text { De acuerdo a la etiqueta acercada al dispositivo móvil se } \\
\text { cambia el mundo en el cual se desarrolla la historia del vi- } \\
\text { deojuego }\end{array}$ \\
\cline { 2 - 3 } & $\begin{array}{l}\text { De acuerdo a la etiqueta acercada al dispositivo móvil se in- } \\
\text { crementan las vidas del personaje, la fuerza, velocidad, etc. }\end{array}$ \\
\hline \multirow{2}{*}{$\begin{array}{l}\text { Escribir etiquetas } \\
\text { NFC con el } \\
\text { dispositivo móvil }\end{array}$} & transporte de & $\begin{array}{l}\text { De acuerdo a la etiqueta acercada al dispositivo móvil se } \\
\text { cambia el transporte en el cual se moviliza el personaje del } \\
\text { videojuego }\end{array}$ \\
\hline \multirow{2}{*}{$\begin{array}{l}\text { Compartir } \\
\text { contenido entre } \\
\text { dispositivos móviles } \\
\text { haciendo uso de } \\
\text { NFC }\end{array}$} & $\begin{array}{l}\text { Contenido en } \\
\text { tiempo real }\end{array}$ & $\begin{array}{l}\text { Desbloqueos } \\
\text { vos móviles con el mismo videojuego instalado }\end{array}$ \\
\cline { 2 - 3 } & $\begin{array}{l}\text { Guardar el estado actual del videojuego en la etiqueta NFC } \\
\text { acercada al dispositivo móvil con el objetivo de iniciar en el } \\
\text { mismo punto en cualquier otro dispositivo móvil que tenga } \\
\text { instalado el videojuego }\end{array}$ \\
\hline $\begin{array}{l}\text { Al acercar a otro dispositivo móvil con el mismo videojuego } \\
\text { pero en un estado más avanzado se puede desbloquear los } \\
\text { mundos o el contenido que éste tiene de más }\end{array}$ \\
\hline
\end{tabular}

Fuente: elaboración propia

\section{CONCLUSIONES}

Una vez realizada la triangulación de las tres fuentes de información: información comercial, tiendas de aplicaciones existentes en el mercado y fuentes bibliográficas respecto al tema general de la investigación, se concluye: 
- En los videojuegos categorizados como interpersonales y/o educativos se hace más adecuado el uso de NFC, debido a que permite implementar videojuegos en estas categorías que mejoren la interacción presencial de los usuarios con objetos o entre ellos mismos, haciendo uso de las etiquetas y otras de sus propiedades para generar contenidos educativos y que requieran la colaboración de varias personas en un mismo lugar.

- Los sistemas operativos en los cuales ha sido desarrollada la mayoría de los videojuegos están cayendo en desuso; esto brinda una oportunidad para innovar en nuevos videojuegos que implementen NFC para los sistemas operativos más utilizados en los mercados actuales.

- La tecnología NFC se ha implementado en varios videojuegos para los siguientes usos: acercar el dispositivo móvil a otro o a las tarjetas de esta misma tecnología para desbloquear nuevo contenido para los juegos, observar el juego en ambas pantallas de los dispositivos móviles utilizados, acercar el dispositivo a las etiquetas NFC que representen el contenido que aparece en el juego, retar a un compañero en modo multi-jugador y utilizar cada etiqueta NFC como un punto de control.

- La metodología de evaluación utilizada permitió llegar a resultados similares por las distintas fuentes; por ejemplo, los dispositivos móviles Nokia han sido los más utilizados para el desarrollo de videojuegos con NFC; esto se explica debido a que muchos de los videojuegos fueron creados varios años atrás, tiempo en el que los dispositivos Nokia eran unos de los pocos que traían incorporada dicha tecnología.

- Algunos de los videojuegos desarrollados con NFC no son muy conocidos por el público, debido a que algunos son diseñados para contribuir únicamente a una investigación específica o no han sido publicados en las tiendas de aplicaciones para dispositivos móviles.

- La concentración de los videojuegos con NFC en investigaciones para sistemas operativos obsoletos se presenta debido al bajo número de dispositivos móviles que implementaban dicha tecnología unos años atrás; en la actualidad se puede avanzar hacia soluciones más modernas dado que dicho número ha estado en constante crecimiento; además, existen nuevas tecnologías que pueden complementar NFC para mejorar la experiencia del usuario y la usabilidad del videojuego.

\section{AGRADECIMIENTOS}

Los autores brindan agradecimientos a la Universidad del Cauca, en especial al Departamento de Telemática por brindar los insumos necesarios para la realización de este artículo. 


\section{REFERENCIAS}

[1] M. Khosrow-Pour, "Video Game Genres", en Encyclopedia of Information Science and Technology, Ed., 2nd ed. Hershey: IGI Publishing, 2008, pp. 3070-3076.

[2] P. Coulton et al., "Experiencing 'Touch' in Mobile Mixed Reality Games", GDTW 2006 The Fourth International Game Design and Technology Workshop and Conference, pp. 68-75, 2009.

[3] C. Crawford, "A Taxonomy of Computer Games", en The Art of Computer Game Design, Ed., Berkeley: McGraw-Hill, 1984, pp. 25-34.

[4] J. L. Campíns, Desarrollo de la lógica de un videojuego de plataformas en Android, 2012.

[5] E. Solomon, “Computer participation in games", en Games Programming, Ed., Cambridge: Cambridge University Press, 1984, pp. 9-32.

[6] D. Johnson et al., "The Edge of Glory: The Relationship Between Metacritic Scores and Player Experience", Proceedings of the First ACM SIGCHI Annual Symposium on Computer-human Interaction in Play, pp. 141-150, 2014.

[7] M. Wolf, "The video game explosion: a history from Pong to Playstation and beyond", Ed., USA: Greenwood Publishing Group, 2008.

[8] E. Adams, “The genres of games”, en Fundamentals of Game Design, Ed., 2nd ed. Berkeley: New Riders, 2009, pp. 390-617.

[9] H. Philip, Java Games Programming, course CSC207.

[10] S. Belli and C. López, "Breve historia de los videojuegos", Athenea Digital, n. 14, pp. 159$179,2008$.

[11] J. Stenros and M. Montola, “Chapter Two - Pervasive Game Genres”, Pervasive Games, Ed. Boston: Morgan Kaufmann, 2009 pp. 31-46.

[12] C. deGraft-Johnson et al., "Relating Five Factor Personality Traits to Video Game Preference", Human-Computer Interaction Technical Report, p. 16, 2013.

[13] J. Fritts, “Computer \& Video Game Genres”, CSCI 130 - Computer Game Design, p. 21.

[14] J. Castromil. (2014, June). Nintendo Amiibo, la Wii U usará figuras reales NFC en sus videojuegos. [En línea], acceso octubre, 2014. Disponible: http://clipset.20minutos.es/ nintendo-amiibo-la-wii-u-usara-figuras-reales-nfc-en-sus-videojuegos/

[15] V. Abarca. (2012, Sept.). NFC: Preguntas y respuestas. [En línea], acceso octubre, 2014. Disponible: http://victorabarca.es/nfc-preguntas-y-respuestas/

[16] ID Noticias. (2014, Feb.). Pronóstico: 400 \% incremento de ventas NFC en 2018. [En línea], acceso octubre, 2014. Disponible: http://www.idnoticias.com/2014/02/13/ pronostico-400-incremento-de-ventas-nfc-en-2018 
[17] ABC Tecnología. (2013, Oct.). La tecnología NFC convierte las mesas de McDonald's en circuitos de carreras. [En línea], acceso octubre, 2014. Disponible: http://www.abc.es/tecnologia/ moviles-aplicaciones/20130830/abci-mcdonalds-eses-carreras-201308301941.html

[18] Manuls. (2011, June). Angry Birds Magic traerá el NFC para los juegos. [En línea], acceso octubre, 2014. Disponible: http://www.xatakaon.com/dispositivos-multimedia/ angry-birds-magic-traera-el-nfc-para-los-juegos

[19] A. Carbajo. (2014, Jan.). Más muestras del potencial de iBeacon, videojuegos para iOS. [En línea], acceso octubre, 2014. Disponible: http://www.applesfera.com/curiosidades/ mas-muestras-del-potencial-de-ibeacon-videojuegos-para-ios

[20] M. Peláez. (2014, June). La Tecnología iBeacon se Usará en un Juego Promocional durante el CES. [En línea], acceso octubre, 2014. Disponible: http://www.ipadizate.es/2014/01/05/ ibeacon-juego-ces-81334/

[21] Laneterralever. (2013, Dec.). iBeacon: The Underrated, Game-changing Technology of 2014. [En línea], acceso octubre, 2014. Disponible: http://www.laneterralever.com/ ibeacon-technology-changing-marketing-in-2014/

[22] S. Munoz-Quintero et al., "NFC Assessment for Video Games on Mobile Devices", Memorias del VII Congreso Iberoamericano de Telemática CITA2015, pp. 89-96, 2015.

[23] Microsoft. Soporte, dispositivos móviles. [En línea], acceso octubre, 2014. Disponible: http:// www.microsoft.com/es-xl/movil/soporte/producto/nokia-store-transition/

[24] D. Christin et al., "CachedSensing: Exploring and documenting the environment as a treasure hunt”, Local Computer Networks Workshops (LCN Workshops), pp. 973-981, 2012.

[25] O. Rashid et al., "PAC-LAN: Mixed-reality Gaming with RFID-enabled Mobile Phones", Computers in Entertainment (CIE) - 3rd anniversary issue, Vol.4, n. ${ }^{\circ}$ 4, 2006.

[26] O. Rashid et al., "Utilising RFID for mixed reality mobile games”, ICCE '06. 2006 Digest of Technical Papers, pp. 459, 460, 2006.

[27] E. Siira and V. Törmänen, "The Impact of NFC on Multimodal Social Media Application”, Near Field Communication (NFC), 2010 Second International Workshop, pp. 51-56, 2010.

[28] J. Haikio et al., “'Would You Be My Friend?' - Creating a Mobile Friend Network with 'Hot in the City", System Sciences (HICSS), 2010 43rd Hawaii International Conference, pp. 1-10, 2010 .

[29] A. Nandwani et al., "NFC Mobile Parlor Games Enabling Direct Player to Player Interaction", Near Field Communication (NFC), 2011 3rd International Workshop, pp. 21-25, 2011.

[30] G. Broll et al., "Touch to Play: Mobile Gaming with Dynamic, NFC-based Physical User Interfaces", Proc. the 12th International Conference on Human Computer Interaction with Mobile Devices and Services, (MobileHCI '10), pp. 459-462, 2010. 
[31] G. Broll et al., "Touch to Play -- Exploring Touch-Based Mobile Interaction with Public Displays," Near Field Communication (NFC), 2011 3rd International Workshop, pp. 15-20, 2011.

[32] E. Di Rosa and F. Benente, “"Excavate and learn": Enhancing visitor experience with touch and NFC,” Digital Heritage International Congress (DigitalHeritage), vol.2, pp. 689-692, 2013.

[33] L .Sarmenta, "Tangible and Casual NFC-Enabled Mobile Games", Pervasive Computing, vol 7319, pp. 361-369, 2012.

[34] A. Lucero et al., "The Playful Experiences (PLEX) Framework As a Guide for Expert Evaluation", Proceedings of the 6th International Conference on Designing Pleasurable Products and Interfaces, 2013.

[35] M. Wolbert et al., "CountMeIn: Evaluating Social Presence in a Collaborative Pervasive Mobile Game Using NFC and Touchscreen", Proceedings of the 11th Conference on Advances in Computer Entertainment Technology (ACE '14). article 5, pp. 10, 2013.

[36] M. Wolbert and A. El Ali, "Evaluating NFC and Touchscreen Interactions in Collaborative Mobile Pervasive Games", Proceedings of the 15th International Conference on Humancomputer Interaction with Mobile Devices and Services, (MobileHCI ‘13), pp. 522-527, 2013.

[37] A. Gentes and C. Jutant, "Pervasive Gaming: Testing Future Context Aware Applications", Journal COMMUNICATIONS \& STRATEGIES, n. ${ }^{\circ}$ 73, pp. 81-103, 2009.

[38] M. Simatic, and A. Gentes, "RFID-based Distributed Shared Memory for Pervasive Games", Mobile Computing, Applications and Services, vol. 35, pp. 339-342, 2010.

[39] E. de la Guía et al., "Interacting with Objects in Games Through RFID Technology", Radio Frequency Identification from System to Applications, p. 166, 2013.

[40] A. Koivisto et al., "Combining the Dimensions of Written and Digital Media in a NFC-based Non-linear Adventure Game for Children", IFLA WLIC 2014 - Lyon - Libraries, Citizens, Societies: Confluence for Knowledge, 2014.

[41] M. Ervasti et al., "Experiences from NFC Supported School Attendance Supervision for Children", Mobile Ubiquitous Computing, Systems, Services and Technologies, UBICOMM '09. Third International Conference, pp. 22-30, 2009.

[42] L. Murmann et al., "NFC Heroes - Observing NFC Adoption through a Mobile Trading Card Game", Research in the LARGE, LARGE 3.0, pp. 19-22, 2012.

[43] M. Kranz et al., "Research in the Large: Challenges for Large-Scale Mobile Application Research- A Case Study about NFC Adoption using Gamification via an App Store", International Journal of Mobile Human Computer Interaction (IJMHCI), pp. 45-61, 2013. 
